\title{
eJRIEPS
}

Ejournal de la recherche sur l'intervention en éducation physique et sport

$40 \mid 2017$

Varia

\section{Les garçons face à la mixité et à la non mixité en EPS}

\section{Antoine Bréau et Vanessa Lentillon-Kaestner}

\section{OpenEdition}

\section{Journals}

Édition électronique

URL : http://journals.openedition.org/ejrieps/715

DOI : 10.4000/ejrieps. 715

ISSN : 2105-0821

Éditeur

ELLIADD

\section{Référence électronique}

Antoine Bréau et Vanessa Lentillon-Kaestner, «Les garçons face à la mixité et à la non mixité en EPS », eJRIEPS [En ligne], 40 | 2017, mis en ligne le 01 janvier 2017, consulté le 01 août 2019. URL : http:// journals.openedition.org/ejrieps/715; DOI : 10.4000/ejrieps.715

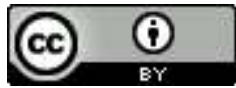

La revue eJRIEPS est mise à disposition selon les termes de la Creative Commons Attribution 4.0 International License. 


\section{Les garçons face à la mixité et à la non mixité en EPS}

Antoine Bréau* \& Vanessa Lentillon-Kaestner*

* Unité d'Enseignement et de Recherche en Education Physique et Sportive, Haute Ecole Pédagogique du canton de Vaud, Suisse

\section{Résumé}

Face au maintien des inégalités entre les sexes à l'école, le retour de la non mixité est une perspective discutée et déjà mise en place dans certains pays. En éducation physique et sportive (EPS), le débat autour d'un enseignement mixte ou non mixte continue d'occuper une place importante. En Suisse, au sein du canton de Vaud, les élèves scolarisés en classe de secondaire 1 (collège) et de secondaire 2 (lycée) connaissent un enseignement mixte et séparé. Cette étude a ainsi souhaité accéder, selon le degré de scolarité et le niveau de réussite en EPS, au point de vue des garçons à propos des cours mixtes et non mixtes en EPS. A partir de la mise en place de 12 focus groups, cet article souligne que la majorité des garçons interrogés (70\%) préfère s'engager au sein d'une EPS non mixte. La non mixité, avant tout valorisée par les élèves de secondaire 1 et ceux affichant un niveau de réussite plutôt élevé en EPS, est notamment plébiscitée car elle permet de s'engager pleinement et sans restriction dans une EPS plus compétitive et où le niveau de pratique est meilleur. La mixité est défendue par une minorité de garçons ; certains d'entre eux, qui présentent plutôt un niveau de réussite moyen ou faible en EPS sont revenus sur leur expérience difficile vécue en contexte séparé. Finalement, cette étude vient questionner le modèle de masculinité susceptible d'être véhiculé par une EPS non mixte, les stéréotypes de genre qui s'y rattachent et la prise en compte de la singularité de chaque élève.

Mots clés: mixité, non mixité, masculinité, adolescence, stéréotypes de genre, focus groups

\section{Introduction}

A une échelle internationale, la question du genre à l'école est devenue un " thème crucial » (Rapport Eurydice, p. 3) notamment au regard de la persistance des inégalités entre les filles et les garçons. Face au maintien des stéréotypes de genre au sein des 


\section{eJRIEPS 40 janvier 2017}

établissements scolaires, l'école continue d'être "embarrassée par la mixité " (Dubet, 2010, p. 78). Critiquée en tant que principe pédagogique, la mixité est remise en cause dans certains pays (e.g., Angleterre, Australie, Canada, Corée du Sud, Etats-Unis) où le nombre d'écoles et de classes non mixtes progresse chaque année (Bigler, Hayes \& Liben, 2014). Le retour de la non mixité à l'école s'inscrit comme une volonté d'améliorer la qualité de l'éducation, à travers un climat de classe plus sain et plus favorable aux apprentissages. La non mixité vise ainsi l'amélioration des performances scolaires des élèves, et notamment celles des garçons, souvent jugés comme étant les principales victimes de la mixité ou encore comme le "nouveau deuxième sexe à l'école " (Auduc, 2009, p. 10). De plus, la séparation des filles et des garçons doit permettre aux élèves de ne pas rester enfermés dans des modèles de féminité et de masculinité déjà préconçus où l'engagement de chacun et de chacune reste dicté par les normes de genre (CrawfordFerre \& Wiest, 2013). Décrite comme une nouvelle forme d'éducation qui veut offrir les mêmes chances et les mêmes opportunités aux deux sexes, la non mixité veut ainsi favoriser un engagement libre et total dans des disciplines souvent stéréotypées comme les sciences ou l'éducation physique et sportive (EPS).

En EPS, le débat autour d'un enseignement mixte ou non mixte occupe toujours une place importante, fréquemment débattue (Berg \& Lahelma, 2010 ; Best, Pearson \& Webb, 2010 ; Hills \& Croston, 2012). Parce qu'on ne saurait «penser le corps sans son sexe, sans la sexuation " (Fraisse, 1996, p. 10), l'EPS est une discipline scolaire directement engagée dans le processus de construction du genre et dans l'égalité entre les filles et les garçons. En impliquant le corps des élèves et en s'appuyant sur des activités sportives traditionnellement vues comme masculines dans lesquelles la différence des sexes ne peut y être neutralisée, l'EPS est souvent considérée comme une arène pour la production de genre, un lieu de construction des différences et des inégalités entre les sexes (Bramham, 2003 ; Fagrell, Larsson \& Redelius, 2012). Proposer une EPS non mixte fait notamment suite aux nombreuses études dressant le portrait d'une discipline qui en situation de mixité participe à la diffusion d'un sexisme caché et à la (re)production des inégalités de genre (e.g., Guérandel \& Beyria, 2010; Koca, 2009). Les inégalités présentes en cours d'EPS mixtes se retrouvent notamment au sein des résultats scolaires des filles, toujours inférieurs à ceux des garçons ou encore au niveau de leur investissement dans les activités physiques et sportives, qui tend à se réduire tout au long de l'adolescence (Dumith, Gigante, Domingues \& Kohl, 2011). Au sein d'un contexte mixte, certaines filles ont ainsi tendance à moins s'engager que les garçons dans les 


\section{eJRIEPS 40 janvier 2017}

situations proposées et à être plus souvent absentes (Azzarito, Solmon \& Harrison, 2006 ; With-Nielsen \& Pfister, 2011). La domination masculine en EPS s'exprime également au sein des interactions entre les enseignants et les élèves, et des enseignants qui vont globalement passer plus de temps avec les garçons et qui vont avoir des attentes moins élevées envers les filles (Couchot-Schiex \& Trottin, 2005). Une autre part du sexisme caché se présente lorsque les enseignants privilégient avant tout les garçons pour réaliser les démonstrations en cours (Castillo-Andrès, Romera-Granados, Gonzalez-Ramirez \& Campos-Mesa, 2012) ou alors lorsque des expressions stéréotypées sont utilisées pour décrire l'activité des filles (Rǿnholt, 2002). En contexte mixte, une certaine "loi des garçons " est bien présente (Patinet-Bienaimé \& Cogérino, 2011, p. 3), avec des enseignants d'EPS qui tendent à organiser et à gérer la classe en prenant comme référence majeure le comportement des garçons.

Si une large majorité des études menées s'est intéressée aux difficultés rencontrées par les filles en EPS, la question des expériences vécues par les garçons interroge (Cogérino, 2005 ; Thorne, 1993 ; Tischler \& McCaughtry, 2011). A l'instar de certaines adolescentes, des garçons, moins en réussite dans cette discipline, peuvent aussi se sentir exclus au sein d'une EPS qui valorise uniquement le modèle d'un corps fort, puissant, musclé et compétitif (Hickey, 2008). A travers la prédominance des activités traditionnelles et des sports de compétition programmés en cours d'EPS (Combaz \& Hoibian, 2008), les élèves sont en effet confrontés à l'idéal d'un corps performant et à une définition précise de la masculinité. Le plus souvent organisés autour de la puissance physique, de l'agressivité ou encore de l'affrontement, les apprentissages proposés en EPS participent à la diffusion d'une masculinité hégémonique, qui se caractérise par la recherche de vitesse, l'acceptation du risque, la mentalité de vainqueur ou encore le manque d'empathie (Bramham, 2003 ; Martino \& Berrill, 2003). La masculinité hégémonique, qui valorise une certaine forme de virilité, tend à définir la manière la plus valorisée d'être un homme, par rapport à laquelle tous les hommes doivent se positionner (Connell \& Messerschmidt, 2005). En tant que discipline scolaire, l'EPS occupe une place particulière dans la construction de la masculinité dans la mesure où, au cours de l'adolescence, le corps s'inscrit comme un vecteur d'intégration de la virilité contemporaine avec des garçons encouragés à affirmer leur force, leur pouvoir, leur courage mais aussi leur hétérosexualité (Connell, 2002 ; Duret, 1999; Guérandel, 2013). En EPS, la diffusion du modèle hégémonique de la masculinité ou encore d'une « hypermasculinité " tend à négliger la présence d'autres formes de masculinités et à exclure des adolescents moins attirés vers 


\section{eJRIEPS 40 janvier 2017}

les valeurs de compétition et de performance (Hickey, 2008 ; Tischler \& McCaughtry, 2011). Au sein des cours d'EPS, Garcia (2000) décrit ainsi « le mal être d'adolescents peu performants, qui se voient confrontés directement aux compétences et aux corps des autres, modèles référents, qui eux ne craignent ni la compétition, ni le regard de l'autre car ils répondent à la norme d'excellence, norme établie du social » (p. 13). Face à la présence de ces garçons qui souffrent en EPS, ou plus généralement à l'école, l'étude de la construction sociale de la masculinité au sein de classes mixtes et non mixtes se poursuit, à l'instar de travaux qui privilégient l'expérience vécue par les garçons au sein de l'institution scolaire (e.g., Ayral \& Raibaud, 2014 ; Welzer-Lang, 2010 ; Welzer-Lang \& Zaouche-Gaudron, 2011)

En EPS, les études menées sur la non mixité restent limitées (Hannon \& Williams, 2008). Les principaux travaux réalisés se sont surtout intéressés à la question des filles, qui en contexte séparé tendent à se sentir plus à l'aise et moins gênées par les garçons (Ollis \& Meldrum, 2009). A partir d'un climat de classe différent, moins centré sur la compétition, la non mixité peut permettre une meilleure participation des filles et un meilleur apprentissage, loin des moqueries et des remarques des garçons. Ces travaux confirment des discours souvent entendus autour de l'intérêt de proposer certains moments non mixtes en EPS afin de permettre aux filles de se libérer de modèles qui les infériorisent (e.g., Dubet, 2010 ; Fize, 2003). Si la non mixité peut favoriser une meilleure participation des filles, d'autres recherches soulignent toutefois que les stéréotypes de genre restent bien présents et que la séparation entre le masculin et le féminin peut se renforcer (e,g., Berg \& Lahelma, 2010 ; Hill, 2015). Du côté des garçons, les travaux réalisés en contexte non mixte, moins nombreux que pour les filles, insistent sur le renforcement d'un climat de compétition et d'affrontement, pouvant mettre en difficulté et intimider des élèves ayant de moins bonnes performances (Bohler, 2004). Concernant les préférences des élèves en contexte mixte et non mixte, les résultats sont partagés (Hills \& Croston, 2012) : si certaines études soulignent que les garçons et les filles préfèrent s'engager dans un contexte mixte (Combaz, 1991; Gagné, Martel \& Nadeau, 2006 ; Lentillon, 2009b), d'autres expliquent que les élèves préfèrent l'EPS enseignée de manière séparée (e.g., Treanor, Graber, Housner \& Wiegand, 1998). Notons toutefois qu'en fonction de l'âge et du degré de scolarité des élèves (collège, lycée) des différences apparaissent concernant les préférences des élèves vis à vis de la mixité ou de la non mixité en EPS : les collégiens sont ainsi plus favorables à la non mixité en EPS que les lycéens (Combaz, 1991 ; Lentillon, 2009b). Les préférences des élèves pour la mixité ou la non mixité 


\section{eJRIEPS 40 janvier 2017}

peuvent également être mises en relation avec le niveau de réussite des élèves au sein de cette discipline scolaire. Par exemple, l'étude menée par Gagné, Martel et Nadeau (2006) souligne que les garçons en difficulté en EPS préfèrent s'engager dans un contexte mixte. Enfin, notons qu'à propos des travaux réalisés sur la comparaison entre la mixité et la non mixité en EPS (mais aussi plus généralement à l'école), des critiques apparaissent concernant la méthodologie employée et le contrôle de la «variable sexe » (Smyth, 2010). D'autres variables (e.g., caractère public ou privé de l'établissement, politique de recrutement des élèves, sélection des enseignants, effectifs des classes) tendent en effet à jouer un rôle non négligeable dans les performances scolaires et les attitudes des élèves. Au-delà des limites méthodologiques, des critiques mentionnent l'importance accordée aux discours et aux anecdotes en lieu et place de recherches scientifiques, notamment qualitatives (Cogérino, 2007 ; Fergus, Noguera \& Martin, 2014).

En Suisse, la mixité n'est pas obligatoire au niveau national. Selon les régions, voire même les établissements, l'enseignement de l'EPS au niveau secondaire connaît des situations très diverses et relève d'une politique locale (choix de l'équipe de direction et/ou de l'équipe enseignante d'EPS). En Suisse romande, au sein des cantons de Genève et de Vaud, les cours d'EPS proposés peuvent ainsi être mixtes ou séparés. Couchot-Schiex et Deriaz (2013) soulignent que « les recherches sur la mixité en éducation physique sont quasi inexistantes à Genève comme d'ailleurs en Suisse Romande » (p. 113). La présence de cours non mixtes au sein des établissements scolaires suisses s'appuie notamment sur la recommandation du 28 octobre 1993 de la conférence des directeurs de l'instruction publique (CDIP) qui souligne qu'il est envisageable de « déroger au principe de la mixité des classes pour autant que l'égalité des deux sexes soit encouragée ». Aujourd'hui, « si cette recommandation garde toute son actualité » (Grossenbacher, 2006, p. 57), c'est notamment parce que des inégalités entre les filles et les garçons à l'école sont dénoncées (Vouillot, 2010).

Au sein de notre étude, nous avons choisi de nous centrer sur le point de vue des élèves, et notamment celui des garçons à propos de l'expérience vécue en contexte mixte et non mixte. Tous les élèves interrogés ont vécu ou vivent encore un enseignement mixte et non mixte en EPS. Dans le canton de Vaud (Suisse), l'EPS s'enseigne au sein de deux (secondaire 2) ou trois périodes (secondaire 1) de 45 minutes hebdomadaires. En secondaire 1 , les élèves peuvent au cours d'une même semaine s'engager dans un contexte mixte et dans un contexte non mixte. Concernant les expériences vécues par les élèves en EPS, notons que l'enseignement de cette discipline au sein des cantons de 


\section{eJRIEPS 40 janvier 2017}

Suisse romande s'est accordé depuis 2010 sur un plan d'études romand (PER) dans lequel l'EPS s'intègre au domaine «Corps et mouvement». En EPS, la mission des enseignants consiste notamment à enrichir le répertoire moteur des élèves, à optimiser leurs ressources physiques et motrices ainsi qu'à développer leur capital santé à partir d'apprentissages réalisés dans des situations variées, tant dans le domaine du mouvement que dans l'expression corporelle. Ces apprentissages doivent également contribuer à la « formation générale » des élèves (e.g., Santé et bien-être, vivre ensemble et exercice de la démocratie) et au développement des « capacités transversales » (e.g., démarche réflexive, collaboration, communication). Concernant la prise en compte de l'égalité entre les sexes au sein de ces programmes, elle reste limitée avec la seule description d'un élève générique et asexué (Fassa, 2013).

En laissant la parole aux élèves, l'objet de ce travail était de repérer, selon le niveau de scolarité (secondaire 1 et 2 ) et le niveau de réussite en EPS, les préférences de chacun en fonction du contexte et de rendre compte des différents avantages ou inconvénients posés par la mixité et la non mixité. Jugés comme particulièrement efficaces pour explorer le point de vue des enfants et des adolescents (Morgan, Gibbs, Maxwell \& Britten, 2002), des focus groups ont ainsi été mis en place avec les adolescents.

\section{Méthode}

2. 1. Echantillon

Dans cette étude, 46 garçons ont été interrogés. 36 étaient scolarisés en secondaire 1 (collège) et 10 en secondaire 2 (lycée). En Suisse, le secondaire 1 fait suite à l'école primaire et dure trois ans. Avec lui s'achève l'obligation scolaire. Ensuite, le secondaire 2 représente la première phase de la scolarité post-obligatoire. Les élèves interrogés étaient âgés de 12 à 19 ans $(\mathrm{M}=15.04, \mathrm{ET}=1.97)$. Ils étaient tous volontaires pour participer à cette étude, suite à un appel à participation lancé dans différentes classes d'établissements de secondaire 1 et de secondaire 2.

2. 2. Outils de recueil de données

Privilégiant une approche qualitative, nous avons mis en place des focus groups auprès d'élèves. Ces « entretiens de groupe », qui reposent avant tout sur la communication et les interactions ont été réalisés à partir d'un guide d'entretien afin de susciter un débat d'idées chez les participants. La grille d'entretien regroupait d'une manière générale différentes questions ouvertes autour du rapport que les élèves ont avec les cours d'EPS (e.g., «Comment appréciez-vous les cours d’EPS? Ressentez-vous du plaisir en 


\section{eJRIEPS 40 janvier 2017}

EPS ?"), les différentes activités enseignées (e.g., "Quelles activités aimez-vous pratiquer en EPS ? »), ou encore leurs préférences vis à vis d'un contexte mixte ou non mixte en EPS (e.g., " Préférez-vous pratiquer l'EP filles / garçons mélangés, séparés ou ça vous est égal ? ", "Comment cela se passe quand vous êtes séparé(e)s en EPS ? mélangé(e)s ?»). Au-delà des seules préférences, les élèves étaient également invités à faire part des différents avantages ou inconvénients propres à chaque contexte. Une fiche de renseignement a été distribuée à chaque participant notamment pour avoir le niveau de réussite perçu en EPS («Quel est ton niveau en EPS : faible, moyen ou fort ? »). Dans cet article, nous nous sommes uniquement focalisé sur le point de vue des garçons à propos de l'enseignement de l'EPS en contexte mixte ou séparé.

\section{3. Procédure}

Au total, 12 focus groups ont été réalisés auprès d'élèves du canton de Vaud en Suisse, dans six établissements de secondaire 1 (9 focus groups) et deux établissements de secondaire 2 (3 focus groups). Ces focus groups ont été menés de février 2014 à janvier 2015 et ont tous été intégralement filmés. Ils se sont déroulés pendant les heures d'école, dans une salle de classe choisie par l'animateur, et dans une atmosphère détendue. Sur les 12 focus groups réalisés, certains étaient mixtes $(n=5)$ et d'autres ne regroupaient que des garçons $(n=7)$. Le respect de l'anonymat et de toutes les opinions a été précisé en début de séance. La durée de chaque focus group était comprise entre 45 minutes et une heure. Pour chaque entretien collectif, un nombre suffisamment important d'élèves était convoqué (entre quatre et six élèves) afin de favoriser les interactions (Kitzinger, Markova \& Kalampalikis, 2004). Deux chercheurs étaient systématiquement présents au cours de l'entretien (un animateur et un observateur). L'animateur avait pour fonction de conduire le focus group et d'encourager les participants à donner leur point de vue, en créant notamment un climat de confiance. II adoptait une attitude d'écoute active, avec attention et sensibilité, et ne cherchait pas à contrôler les questions soulevées. II était également important pour l'animateur de gérer la dynamique de groupe, notamment à travers la prise en compte de tous les élèves, leaders ou alors plus en retrait. Afin que chaque adolescent puisse s'exprimer, un tour de table était systématiquement réalisé dès la première question ainsi que lorsque les élèves devaient préciser leurs préférences vis à vis de la mixité ou de la non mixité. En fonction des focus groups et des discussions menées entre les élèves, l'animateur pouvait également distribuer la parole pour assurer une participation de tous les adolescents et pour conserver l'idée d'un débat collectif et partagé. Au cours des débats, l'animateur pouvait aussi prendre soin de ramener le débat 


\section{eJRIEPS 40 janvier 2017}

vers le sujet principal et d'aborder les différents thèmes ou sous-thèmes préparés (e.g., climat de classe, motivation, apprentissages). A travers certaines relances, des précisions pouvaient être demandées afin d'approfondir les débats et d'ouvrir une réflexion plus large (e.g., préférence mixité/non mixité selon le type d'activité pratiqué). L'observateur était présent pour accueillir les participants, gérer les aspects techniques (enregistrement) et prendre des notes sur le déroulement de l'entretien, les interactions et les éléments « clés » évoqués, susceptibles d'être utilisés lors de l'analyse thématique. II a veillé à rester neutre et n'a pas participé aux discussions. A l'issue des focus groups, un bilan (oral puis écrit) était directement réalisé par l'animateur et l'observateur. L'objectif était à la fois de réaliser une synthèse rapide des différentes thématiques abordées par les élèves mais aussi des difficultés rencontrées lors de la réalisation du focus group.

\section{4. Analyse des données}

Les entretiens et l'analyse des données ont été réalisés par le même chercheur. Les enregistrements ont été retranscrits dans leur intégralité. Les transcriptions verbatim ont été lues plusieurs fois afin d'identifier et de sélectionner les données en lien avec la mixité et la non mixité en EPS. Une analyse de contenu thématique de type inductif et déductif (thèmes abordés dans la grille d'entretien et thèmes émergents) a ensuite été effectuée afin de faire ressortir différents thèmes reliés à la question de la mixité et de la non mixité en EPS (Mucchielli, 1998). Trois grands thèmes ont ainsi émergé : les préférences des garçons à propos de la mixité et de la non mixité en EPS, les avantages de la non mixité en EPS et enfin, les avantages liés à un contexte mixte. Les réponses des élèves ont tout d'abord été analysées en général puis en comparant les réponses des élèves scolarisés en secondaire 1 et en secondaire 2 ainsi qu'en fonction du niveau de réussite en EPS. A propos du niveau de réussite en EPS, les réponses obtenues au sein de la fiche de renseignement ont permis d'identifier trois groupes: le niveau fort $(n=20)$, le niveau moyen $(n=24)$ et le niveau faible $(n=2)$. En fonction de ces deux variables, des différences ont été relevées aussi bien au niveau des préférences des élèves pour la mixité ou la non mixité qu'au niveau des arguments et des inconvénients propres à chaque contexte. Notons également qu'aucune différence n'a été relevée concernant les réponses données par les garçons au sein des focus groups mixtes et non mixtes.

\section{Résultats}

3. 1. Des garçons plutôt en faveur de la non mixité en EPS : des préférences notamment affichées par les élèves de secondaire 1 et ceux en réussite 


\section{eJRIEPS 40 janvier 2017}

Sur les 46 adolescents interrogés au sein des focus groups, tous se sont prononcés concernant leur préférence vis à vis d'un contexte mixte ou séparé en EPS. Près de $70 \%$ $(n=32)$ ont déclaré qu'ils préféraient s'engager en EPS dans un contexte non mixte. Seulement $30 \%(n=14)$ ont manifesté leur préférence pour la mixité. A noter que la non mixité est surtout valorisée par les élèves de secondaire $1(75 \%, n=27)$ alors que le constat est plus mitigé chez les élèves de secondaire $2(50 \%, n=5)$. La non mixité est surtout défendue par les élèves qui considèrent avoir un niveau de réussite élevé (95\%, $n$ = 19) ou moyen $(54 \%, n=13)$. A l'inverse, la mixité est plutôt défendue par les élèves jugeant leur niveau en EPS comme étant faible $(100 \%, n=2)$, voire moyen $(46 \%, n=11)$. Seul un élève ayant répondu avoir un niveau élevé (5\%) a affiché des préférences pour la mixité.

\section{2. Les arguments en faveur de la non mixité en EPS}

Au sein des discussions collectives menées avec les garçons, cinq arguments en faveur de la non mixité en EPS ont pu être relevés: un meilleur niveau de pratique entre garçons, la possibilité de s'investir à $100 \%$ en EPS, un climat de classe centré sur la compétition et l'affrontement, les difficultés posées par la motivation des filles en contexte mixte, et les goûts différents vis à vis des activités physiques et sportives affichés par les deux sexes.

3. 2. 1. La non mixité en EPS valorisée par les garçons car synonyme d'un meilleur niveau de pratique

En EPS, la non mixité a notamment été défendue par les garçons car elle favorise selon eux un meilleur niveau de pratique et d'apprentissage. Sur les 32 élèves favorables à la non mixité, 24 garçons (75\%) ont relevé l'importance de se retrouver entre eux afin de pouvoir s'engager dans un jeu de meilleure qualité. Cet argument a notamment été développé par les élèves qui considèrent avoir un niveau élevé de réussite en EPS (80\%, $\mathrm{n}=16)$, alors qu'il a été moins fréquent chez les élèves de niveau moyen $(33 \%, n=8)$. Aucun élève de niveau faible n'a évoqué cet argument. La question d'un meilleur niveau de pratique au sein d'une EPS non mixte est un argument partagé à la fois au niveau secondaire $1(55 \%, n=20)$ et au niveau secondaire $2(40 \%, n=4)$. En contexte mixte, Eric (secondaire 1, fort en EPS) souligne que «les inconvénients avec les filles, c'est la différence de niveau entre les garçons et les filles ». Ce constat est souvent partagé puis repris par les adolescents, comme Thomas (secondaire 1, moyen en EPS) qui témoigne qu'il est « un peu contre la mixité parce que en fait, il y a une différence de niveau et c'est un fardeau pour ceux qui se donnent la peine et tout ». Dans le discours des garçons, la 


\section{eJRIEPS 40 janvier 2017}

constitution des groupes lors des situations de jeu est notamment évoquée, ces derniers regrettant l'obligation de faire des équipes mixtes et équitables : "L'idéal c'est de séparer garçons et filles parce que comme ça on serait plus à l'aise. Je préfère quand c'est pas mixte. Après on doit faire des équipes équitables, ça joue moins bien " (Jacques, secondaire 1, fort en EPS). En classe de secondaire 2, Samuel confirme ce point de vue avec, en contexte non mixte, "un jeu qui va plus vite et la qualité est meilleure " (secondaire 2, fort en EPS). Lors d'un focus group, Christophe (secondaire 1, fort en EPS) justifiera ainsi son intérêt pour la mixité par une "question de performance ".

3. 2. 2. La possibilité de s'investir à $100 \%$ dans un contexte non mixte : un argument non négligeable pour les élèves de secondaire 1

La non mixité en EPS est également valorisée par une majorité de garçons car elle leur permet de se retrouver entre eux et de pouvoir s'engager à $100 \%$, sans devoir se contrôler face aux filles. Au total, $71 \%(n=23)$ des élèves favorables à la séparation des sexes ont partagé cet avis. Si cet argument a aussi bien été formulé par les élèves forts $(60 \%, n=12)$ que moyens $(46 \%, n=11)$, notons qu'il a uniquement été relevé par des élèves scolarisés en classe de secondaire $1(63 \%, n=23)$. Ainsi, si Lucas (secondaire 1 , moyen en EPS) « n'aime pas trop faire avec les filles, même si certaines ont notre niveau, c'est juste une question qu'en sport, on peut aussi se défouler, apprendre, se dépenser à fond. Et avec les filles, on ne peut pas faire la même chose qu'entre garçons. Je préfèrerais vraiment une classe où il n'y a que des garçons pour bien se dépenser à fond ». Le jeune adolescent poursuit et explique ainsi que « devant une fille, on doit être plus respectueux, elles vont peut-être prendre les choses mal. Entre garçons on se connaît déjà bien et puis on a cette notion de déconner. Enfin les autres garçons ils savent ce que c'est déconner et les filles seront plus sensibles à ce que l'on dit ". L'obligation de devoir se contrôler devant les filles est un argument souvent repris par les garçons. Lucas souligne par exemple que " moi personnellement j'ai pas envie de faire mal à une fille. Il y a des choses que nous faisons entre garçons, courir, se sauter dessus, qu'on ne ferait pas aux filles ». Lucien (secondaire 1, fort en EPS) lui explique " que tous les jours, elles nous disent de ne pas tirer fort ». Pour Julien (secondaire 1, fort en EPS), la non mixité en EPS permet ainsi aux garçons " de vraiment jouer. Entre garçons on peut vraiment être dans le jeu et pis être à fond dedans ». Dans les discours tenus par les élèves, des adolescents ont notamment fait référence aux problèmes posés par les différences morphologiques et physiques entre les deux sexes, des différences qui empêchent un investissement total des garçons : "Quand on fait que avec les garçons, les deux heures c'est plus physique, 


\section{eJRIEPS 40 janvier 2017}

on fait plus de choses » (Julien, secondaire 1, fort en EPS) ; « Les filles ça désavantagent parce qu'elles sont moins physiques » (Guillaume, secondaire 1, fort en EPS). Lors d'une relance de la part du chercheur sur les objectifs proposés par l'EPS et notamment le travail autour du contrôle de soi, Guillaume (secondaire 1, fort en EPS) avouera que " c'est vrai que c'est important d'apprendre à se maîtriser. Aussi l'éducation physique ça a un rôle d'éducation mais je pense quand même que la semaine quand on vient en éducation physique, on vient aussi pas mal pour se dépenser et se défouler, tout en restant dans le domaine de l'école et de l'éducation ».

3. 2. 3. Un climat de classe centré sur la compétition et l'affrontement chez les garçons ; un argument important pour les élèves de niveau fort et moyen en EPS A propos des préférences des garçons pour la non mixité, $71 \%$ des adolescents $(n=23)$ expliquent qu'une EPS qu'entre garçons permet le maintien d'un climat de classe avant tout centré sur la compétition et l'affrontement. Cet argument a uniquement été évoqué par des élèves de niveau fort en EPS $(55 \%, n=11)$ et de niveau moyen $(50 \%, n=12)$. Aucune différence n'est toutefois ressortie en fonction du degré de scolarité des élèves, ce thème étant partagé par les élèves de secondaire $1(52 \%, n=19)$ et de secondaire 2 $(40 \%, n=4)$. Pour Jacques (secondaire 1, fort en EPS), « quand on est entre gars, il y a plus de challenge " et il y a souvent " une ambiance très lourde de compétition ». Dans un autre focus group, Paul (secondaire 1, moyen en EPS) reprend ces arguments et souligne "qu'il y a plus de compétition entre garçons, on veut voir qui est le meilleur chez les garçons. Tandis qu'avec les filles on rigole un peu ». Si la compétition en contexte non mixte occupe une place privilégiée, elle est aussi recherchée : "J'aime bien quand il y a de la compétition entre gars, ça me motive » (Samuel, secondaire 2, fort en EPS). L'esprit de compétition se retrouve aussi lorsque les élèves évoquent l'importance de se mesurer à l'autre et de le défier, quelque chose de plus difficile lorsque les garçons sont avec les filles. Pour Thomas (secondaire 1, moyen en EPS), " si on marque contre une fille, enfin si on dribble une fille au foot, on est tous d'accord pour dire qu'elles ont un moins bon niveau et si on fait quelque chose contre quelqu'un qui est moins bon, c'est beaucoup moins impressionnant. ».

3. 2. 4. Le problème des filles peu motivées en contexte mixte : une difficulté notamment soulevée par les adolescents en réussite en EPS.

Pour justifier leurs préférences vis à vis de la non mixité, 20 garçons (62\%) vont aussi souligner les difficultés posées par la mixité et notamment le manque de motivation que peuvent afficher certaines filles. Cet argument a prioritairement été défendue par les 


\section{eJRIEPS 40 janvier 2017}

garçons affichant un niveau de réussite élevée en EPS $(75 \%, n=15)$ alors qu'il a été moins relevé par les élèves de niveau moyen $(21 \%, n=5)$ et faible $(n=0)$. Aucune différence n'est apparue entre les élèves de secondaire $1(44 \%, n=16)$ et de secondaire $2(40 \%, n=4)$. Des garçons font ainsi référence à "des filles qui ne font rien du tout " (Jacques, secondaire 1, fort en EPS) et qui «viennent pour ne rien faire et ça, ça désavantage l'équipe où elles sont et ça enlève un peu le rythme du cours de gym " (Luc, secondaire 1, moyen en EPS). En contexte mixte, Samuel (secondaire 2, fort en EPS) évoque notamment la présence de filles dans sa classe qui "sont là pour faire de la figuration et qui ne font aucun effort [...]. Je vois surtout un manque de motivation. Quand on va faire un match, les filles n'ont pas envie d'aller sur le terrain, elles restent remplaçantes ". Ce constat sera partagé par les autres garçons interrogés dans le focus group, et même poursuivi par Julien (secondaire 1, fort en EPS) qui décrit aussi celles " qui sont sur leur téléphone. Dans notre classe c'est presque tout le temps comme ça, elles restent assises. Et pis elles ne veulent pas jouer et pis elles ne se donnent pas la peine ». Finalement, la non mixité est ici défendue à cause du manque d'investissement des filles en EPS. Si des garçons ont reconnu la participation et la réussite de certaines filles en EPS, une plus large majorité a décrit " des filles qui ne sont pas super motivées " (Jean, secondaire 1, moyen en EPS). Pour Guillaume (secondaire 1, fort en EPS) « si sur 100 garçons, il y en a 20 qui ne sont pas motivés, pour les filles c'est l'inverse. ”.

3. 2. 5. Des goûts différents pour les filles et les garçons

Enfin, le dernier argument cité par $62 \%$ des garçons $(n=20)$ favorables à la non mixité en EPS est à mettre en relation avec la présence de goûts différents entre les filles et les garçons pour l'EPS et les différentes activités physiques et sportives pratiquées. Cet argument regroupe à la fois des réponses d'élèves de secondaire $1(44 \%, n=16)$ et de secondaire $2(40 \%, n=4)$, ainsi que de niveau fort $(40 \%, n=8)$ et moyen $(50 \%, n=12)$. Jean explique ainsi qu'il "préfère non mixte parce qu'on n'a pas tellement les mêmes goûts en fait filles-garçons ". En contexte mixte, ces garçons regrettent ainsi de perdre du temps lorsqu'ils doivent choisir une activité commune. L'exemple du rapport au football est notamment cité : "Quand on est entre garçons on va pouvoir faire du foot et pas les filles, ben après les filles elles vont se plaindre " (Arthur, secondaire 1, moyen en EPS). Les garçons évoquent aussi un rapport à la compétition qui est différent, et « des filles qui vont penser à des trucs plus calmes où elles sont mieux " (Vincent, secondaire 1, moyen en EPS). Au football (et autres sports collectifs) des garçons ont ainsi opposé les activités artistiques (e.g., danse, acrosport, patinage artistique, cirque), des activités envisagées 


\section{eJRIEPS 40 janvier 2017}

avant tout pour les filles: "On n'aime pas la danse. Ce n'est pas vraiment pour nous " (Jacques, secondaire 1, fort en EPS) ; "Le patinage artistique j'ai horreur. Parce que ça fait un peu fille " (Philippe, secondaire 1, moyen en EPS). Pour eux, la non mixité permet de satisfaire les goûts de chacun et de chacune : "Si on est avec les filles, on devrait faire de la danse" (Jacques, secondaire 1, fort en EPS). Enfin, dans un des focus groups réalisé qu'entre garçons, les cinq garçons interrogés expliqueront qu'au-delà des différences de goûts pour les activités physiques, c'est plus largement le rapport à l'EPS en tant que discipline scolaire qui doit être interrogé. Alors que Thomas (secondaire 1, moyen en EPS) explique que "si nous on prend ce cours comme un truc qui nous fait plaisir, elles, elles prennent ça comme des cours ", Lucas (secondaire 1, moyen en EPS) confirme que "franchement en classe si on nous apprend qu'on a congé, les filles elles sont trop contentes. Nous on est déçu ».

\section{3. Les arguments en faveur de la mixité en EPS}

Au sein des focus groups réalisés avec les garçons, trois principaux arguments en faveur de la mixité sont ressortis : la possibilité de s'engager dans une EPS non compétitive, le maintien d'une meilleure ambiance et d'une identité de classe en contexte mixte ainsi que la présence d'une certaine violence en contexte séparé. Notons que ces différents arguments ont été avancés par ceux préférant la mixité en EPS mais aussi par certains garçons qui malgré leur préférence pour la non mixité, ont également reconnu des avantages propres à la mixité en EPS.

3. 3. 1. Être avec les filles en EPS : un jeu sans compétition pour les garçons, notamment pour les élèves forts, scolarisés en secondaire 1

A propos des avantages liés à la pratique mixte en EPS, 76\% des garçons interrogés ( $n=$ 35) ont évoqué la possibilité de s'engager dans une EPS moins compétitive, plus centrée sur le jeu et l'amusement. Ce constat est d'autant plus vrai pour les élèves de secondaire $1(86 \%, n=31)$, plus nombreux que les secondaire 2 à citer cet argument $(40 \%, n=4)$. En rapport avec le niveau de réussite en EPS, ce sont plutôt les élèves forts qui se sont exprimés sur ce sujet $(85 \%, \mathrm{n}=17)$ même si les élèves de niveau moyen $(71 \%, \mathrm{n}=17)$ et faible $(50 \%, n=1)$ ont également fait part d'une pratique plus détendue en contexte mixte. Avec les filles, l'EPS tend ainsi à être perçue comme un moment de détente et de jeu, un moment moins sérieux qu'entre garçons. Pour ceux qui préfèrent l'EPS en contexte mixte, être avec les filles « c'est cool, c'est plus marrant et c'est plus amical » (André, secondaire 1, fort en EPS). Pour Maxime (secondaire 1, moyen en EPS), "ça fait un moment ensemble où on rigole ". Des arguments similaires seront avancés par les élèves plutôt 


\section{eJRIEPS 40 janvier 2017}

pour la non mixité. Guillaume (secondaire 1, fort en EPS) explique ainsi que la mixité, " c'est plus détente, c'est plus drôle avec les filles, on se marre plus et puis entre garçons c'est plus la compétition, celui qui gagne ». Les garçons interrogés tendent ainsi à définir une EPS différente en fonction de son contexte mixte et non mixte, une EPS qui privilégie plutôt le jeu et la bonne humeur avec les filles. Pour Christophe (secondaire 1, fort en EPS), défenseur de la non mixité en EPS, l'idéal serait une programmation « d'une heure avec les filles et deux heures juste avec les garçons. Comme ça on a un peu de tout, on rigole, on est plus détendu et après c'est vraiment le sport ".

3. 3. 2. Une meilleure ambiance et une identité de classe en contexte mixte : un constat notamment soulevé par les élèves de secondaire 2

Sur les 14 garçons qui préfèrent l'EPS en contexte mixte, 64\% d'entre eux $(n=9)$ vont souligner la présence d'une meilleure ambiance en classe lorsque les garçons et les filles sont regroupés. Cet argument a notamment été soulevé par les élèves scolarisés en classe de secondaire $2(50 \%, n=5)$ et moins par les élèves de secondaire $1(11 \%, n=4)$. Des élèves de chaque groupe de niveau se sont exprimés sur ce sujet avec toutefois des réponses relativement moindres chez les élèves de niveau fort $(10 \%, n=2)$ par rapport au niveau moyen $(25 \%, n=6)$ et faible $(50 \%, n=1)$. Pour Maxime (secondaire 1 , moyen en EPS), « la gym avec les filles ça fait une méga ambiance ». Dans un autre focus group, Adrien (secondaire 1, moyen en EPS) souligne qu'il est d'autant plus important d'être en mixte " car si on a des amies filles, on peut rigoler aussi avec elles à la gym. Si on n'avait que des amis garçons ça serait moins cool ». Au-delà du rapport d'amitié entre les garçons et les filles, des élèves vont aussi défendre l'importance de conserver le groupe classe en cours d'EPS : "Puisqu'on est tous ensemble ça crée une meilleure ambiance " (André, secondaire 1, fort en EPS), " Je préfère mixte, être tous ensemble. Etre avec la classe quoi " (François, secondaire 2, fort en EPS). Pour les élèves interrogés, la mixité en EPS permet de conserver la dynamique et l'identité de la classe. Pour Adrien (secondaire 1, moyen en EPS), être en classe pendant les cours d'EPS est d'autant plus important car cela permet "de s'amuser et de faire des activités ensemble, alors qu'à l'école c'est plus du boulot ».

3. 3. 3. La présence de violence en contexte non mixte : une réalité décrite par les élèves de secondaire 1

Si la non mixité en EPS est plutôt privilégiée par la majorité des garçons interrogés, 16 des 46 adolescents interrogés (34\%) reconnaissent la présence de conflits et le développement d'une violence qui peut apparaître pendant les cours d'EPS entre garçons. 


\section{eJRIEPS 40 janvier 2017}

L'ensemble des réponses données regroupe uniquement des élèves scolarisés en classe de secondaire $1(44 \%, n=16)$. La présence de violence au sein d'une EPS non mixte est un argument partagé aussi bien pour les élèves forts $(30 \%, n=6)$, moyens $(37,5 \%, n=9)$ et faibles $(50 \%, n=1)$. Dans un des focus groups réalisé qu'entre garçons, Jean (secondaire 1, moyen en EPS) souligne ainsi que « certains se ramassent des moqueries car il y a certains clichés qui disent que forcément les garçons savent jouer au foot. Et beaucoup de gens suivent les clichés ». Pour Jacques (secondaire 1, fort en EPS), moins à l'aise en football, le constat est similaire : "Si on fait du foot et qu'on rate, on se fait engueuler». Les autres garçons ont partagé ce point de vue, Luc (secondaire 1, moyen en EPS) rappelant « qu'entre garçons ça peut finir vraiment très mal », « c'est agressif ». Cette violence tend à ce titre à dépasser le simple moment du cours d'EPS pour se retrouver aussi dans les vestiaires : «Ca peut être brutal, par exemple le foot ils vont jouer comme s'ils étaient à la coupe du monde. Dans les vestiaires aussi. Si toute l'équipe qui a perdu est dans le vestiaire, ça commence à s'engueuler " (Jérôme, secondaire 1, fort en EPS).

\section{3. 4. En secondaire 1, le cas de deux garçons exclus en EPS}

Sur les 46 garçons interrogés, deux d'entre eux nous ont fait part d'une violence, voire d'une exclusion pendant les cours d'EPS. Scolarisés en classe de secondaire 1 et affichant un niveau moyen de réussite en EPS, ces deux élèves sont notamment revenus sur des expériences difficiles vécues en EPS. Pierrick (secondaire 1, moyen en EPS) rapporte ainsi «qu'à chaque fois qu'on fait un match, je joue quasiment jamais. Déjà parce qu'ils me font pas de passe mais je me dis tant mieux comme ça je me fais pas engueuler. Je préfère avec les filles parce que je trouve qu'on se fait plus accepter ». II poursuit : “C'est moins marrant avec les garçons parce qu'avec eux si on loupe une balle ou quelque chose comme ça et ça part direct, et il y tout le monde sur quelqu'un ». Plus loin, Pierrick expliquera qu'au sein des jeux, il doit toujours occuper le même poste : « $A u$ lieu de nous mettre en défense ou quelque chose comme ça, non vous rester coller aux caissons et aux goals pour éviter que les buts entrent ». Pour Quentin (secondaire 1, moyen en EPS), entre garçons « on n'apprend rien. Enfin moi personnellement je n'apprends rien les mardis avec les garçons parce que si j'essaie de faire quelque chose, je me fais tout de suite engueuler parce que je me suis raté alors que le lundi après-midi si je me rate, ben c'est pas très grave, elles m'engueulent un petit coup mais c'est pour rigoler ». Quentin souhaite notamment rappeler que certains garçons « crient et menacent de taper ». II affiche ainsi ses préférences pour un contexte mixte, un contexte où « avec 


\section{eJRIEPS 40 janvier 2017}

les filles on rigole et on peut faire beaucoup de choses » ; "On se sent mieux parce qu'on sait qu'on va moins se faire engueuler et tout cas pas agresser. On se sent mieux, on a plus de plaisir ».

\section{Discussion}

Dans notre étude, une majorité des garçons interrogés est plutôt en faveur de la présence d'une EPS non mixte au sein des établissements scolaires. Ces résultats confirment d'autres travaux anglo-saxons qui ont souligné que les élèves, d'une manière générale, préféraient s'engager dans une EPS non mixte (e.g., Treanor, Graber, Housner \& Wiegand, 1998). A l'inverse, les résultats de cette étude s'opposent à des travaux réalisés en France (e.g., Combaz, 1991 ; Lentillon, 2009b) au sein desquels les garçons affichaient une préférence pour une EPS mixte. Notons que contrairement au modèle français où l'enseignement de l'EPS est avant tout mixte, les adolescents suisses interrogés connaissent la non mixité et vivent des cours séparés. Les élèves interrogés ont ainsi pu faire part de leur expérience vécue en contexte mixte et non mixte, une expérience qui commence dès leur entrée en secondaire 1 (collège).

A propos des préférences affichées par les élèves, des différences selon le niveau de scolarité (secondaire 1 / secondaire 2) sont apparues avec des élèves de secondaire 1 plus favorables à la non mixité que les élèves de secondaire 2. Les résultats de cette étude confirment ainsi l'attitude plutôt négative de la part des collégiens à l'égard de la mixité, un constat déjà repéré et partagé par d'autres études (e.g., Combaz, 1991 ; Lentillon, 2009b). Les préférences affichées par les collégiens pour la non mixité peuvent notamment s'expliquer à partir des relations parfois difficiles entre les filles et les garçons au moment de l'adolescence. En pleine période de leur vie où l'identification à une catégorie de sexe devient particulièrement prégnante, l'adolescence se caractérise en effet par un certain « repli homolatique » (Rouyer, 2007, p. 132) avec des adolescents qui privilégient plutôt des relations avec les pairs de même sexe. Au sein de leur ouvrage $A$ l'école. Sociologie de l'expérience scolaire, Dubet et Martucelli (1996) décrivent ainsi l'opposition entre les garçons et les filles, une opposition qui certes « n'empêchent ni les amitiés, ni les flirts, ni les amours » mais qui « se déroulent sur un fond de « guerre » des sexes et d'affirmation exacerbée des stéréotypes » (p. 154).

Les préférences des garçons pour la non mixité en EPS s'appuient notamment sur la présence d'un niveau de pratique plus élevé et la possibilité pour eux de s'engager à $100 \%$, sans avoir le besoin de se contrôler face aux filles. La moindre réussite des filles en 


\section{eJRIEPS 40 janvier 2017}

EPS reste ainsi présente dans le discours des garçons, une situation qui rappelle des études soulignant l'échec féminin plutôt que masculin au sein de cette discipline scolaire (e.g., Lyu \& Gill, 2011). De plus, pour les garçons et notamment les élèves de secondaire 1 interrogés, la non mixité permet de dépasser les difficultés liées aux différences morphologiques et physiques entre les deux sexes, des différences qui tendent à devenir plus importantes au cours de l'adolescence (Cazorla, 2011). Ce constat confirme l'étude menée par Combaz (1991) qui soulignait les difficultés rencontrées par la mixité face à la présence de capacités physiques trop inégales entre les deux sexes. L'enseignement de l'EPS en contexte séparé est également vu comme une formule intéressante par les garçons, notamment face au manque de motivation pouvant être affiché par certaines filles en EPS. Les discours prononcés par les garçons à propos des filles qui ne s'engagent pas coïncident avec des études soulignant le désengagement progressif des filles en EPS (e.g., Azzarito, Salmon \& Harrison, 2006 ; With-Nielsen \& Pfister, 2011) et la célèbre formule « elles papotent, ils gigotent » (Davisse, 1999). Finalement, les arguments utilisés par les garçons en faveur de la non mixité rappellent des discours déjà tenus par des enseignants lorsqu'ils décrivent les différents problèmes (différences morphologiques et motivationnelles entre les deux sexes, moindre réussite des filles) posés par la mixité en EPS (Best, Pearson \& Webb, 2010 ; Cogérino, 2007).

Les discours des garçons interrogés autour de l'importance de conserver une EPS non mixte afin de pouvoir s'engager sans restriction au sein d'un climat de classe plutôt orienté vers la compétition et l'affrontement rappellent des travaux qui défendent l'installation de moments non mixtes à l'école et des moments privilégiés entre garçons (e.g., Fize, 2003). Face à une école mixte parfois qualifiée de «biotope féminin duquel aurait disparu tout esprit de compétition et de confrontation » (Vouillot, 2010, p. 71), la mise en place d'une école non mixte attachée aux valeurs de performance et de compétition est parfois défendue (Gurian, Stevens \& Daniels, 2009). Toutefois, la présence de moments séparés en EPS et l'omniprésence possible d'un climat compétitif au sein des classes questionnent les objectifs poursuivis par cette discipline scolaire (formation générale des élèves, compétences méthodologiques et sociales) ainsi que le modèle de masculinité que l'EPS souhaite véhiculer. En privilégiant un climat de classe centré sur la compétition et l'affrontement, les situations vécues en EPS et les choix réalisés par les enseignants (e.g., activités enseignées, constitution des équipes, interventions au sein du jeu) tendent à diffuser le message d'une masculinité unique, virile et hégémonique, un message qui néglige pourtant la prise en compte d'autres formes de masculinité (Connell, 1999). Ici, 


\section{eJRIEPS 40 janvier 2017}

l'EPS des garçons pourrait finalement être comparée à une « maison des hommes » (Godelier, 1996), une maison déjà observée dans des milieux sportifs (Clément \& Louveau, 2013) et dans laquelle les garçons sont invités à affirmer leur force et à incorporer «les gestes, les mouvements, les réactions masculines, tout le capital d'attitudes qui serviront à être un homme »(Welzer-Lang, 2000, p. 117). De plus, la réflexion autour du modèle de masculinité diffusé semble d'autant plus importante face aux témoignages d'élèves qui souffrent et font part de la présence de violence au sein des cours. Les discours tenus par les élèves dans notre étude confirment d'autres résultats soulignant la présence de garçons exclus au sein de cette discipline (Tischler \& McCaughtry, 2011 ; Thorne, 1993). A ce propos, alors que la non mixité est avant tout valorisée par les élèves affichant un niveau de réussite élevée en EPS, elle est moins appréciée voire critiquée par ceux qui présentent un niveau moyen ou faible. Ces résultats font plus largement écho aux travaux réalisés par Gagné, Martel et Nadeau (2006) qui soulignent que la mixité est avant tout privilégiée par les garçons plutôt en difficulté en EPS car elle permet une meilleure participation aux activités. Les témoignages de garçons qui souffrent et qui peuvent se sentir exclus en cours non mixtes interrogent à la fois la prise en compte de l'hétérogénéité chez les garçons en contexte non mixte ainsi que les différents modèles de masculinité. Face à des adolescents en pleine période de construction identitaire, une réflexion sur la programmation des activités, sur les formes de groupements utilisés, sur les compétences méthodologiques et sociales visées ou encore sur le modèle d'évaluation prévu semble nécessaire afin de respecter le développement chez les garçons d'une masculinité non pas idéalisée mais plutôt personnalisée qui favorise la construction d'une identité authentique et singulière (Haywood \& Mac an Ghaill, 2012 ; Swain, 2006).

La construction de la masculinité chez les adolescents en EPS interroge aussi le rapport que chacun peut avoir avec la féminité, et avec le groupe des filles en EPS. Dans cette étude, des stéréotypes de genre sont ressortis dans le discours des garçons, notamment lorsqu'ils ont évoqué des goûts différents pour les activités sportives. Les stéréotypes de genre entendus autour des sports collectifs et des activités artistiques confirment ainsi la construction sexuée de la culture sportive et une séparation des activités en fonction d'un pôle masculin ou féminin (Hardin \& Greer, 2009). Au-delà du classement des activités, les stéréotypes de genre apparaissent aussi lorsque les élèves comparent une «EPS détente » avec les filles à une «EPS compétitive » entre garçons. A l'instar d'autres études (Lentillon, 2009a; Lentillon \& Cogérino, 2005), ce travail met en exergue une 


\section{eJRIEPS 40 janvier 2017}

intériorisation des stéréotypes de genre chez les adolescents interrogés, notamment chez les élèves scolarisés en secondaire 1. De plus, notre étude vient confirmer d'autres travaux qui insistaient déjà sur le maintien des stéréotypes de genre dans un contexte non mixte et sur la séparation toujours plus forte entre le masculin et le féminin (Berg \& Lahelma, 2010 ; Hill, 2015). Pour Léchenet (2014), l'instauration de « temps séparés pendant lesquels les garçons pourraient exprimer, pour les canaliser, leur agressivité et leurs pulsions, reviendrait en quelque sorte à survalider les stéréotypes de la masculinité dominante en les naturalisant » (p. 166). Face à une EPS qui, en Suisse, propose à la fois des moments mixtes et non mixtes, une réflexion autour des rapports entre la masculinité et la féminité semble importante afin de ne pas prolonger une vision où la masculinité se construit en s'opposant et en rejetant la féminité.

Dans cette étude, notons également que des garçons, plutôt en faveur de moments partagés avec l'autre sexe ont insisté sur l'importance de vivre des moments ensemble et de conserver le groupe classe. Notamment défendus par les lycéens, plus sensibles à la présence d'une meilleure ambiance de classe que les collégiens (Combaz, 1991), les arguments présentés défendent ainsi la classe en tant que groupe. Selon les élèves, les heures passées en EPS contribuent ainsi à renforcer la dynamique et l'identité du groupe classe. Des modèles d'enseignement comme Sport education ou Teaching Games for Understanding qui insistent sur l'engagement des élèves dans une expérience collective authentique, où les notions d'équipe et de groupe occupent une place centrale, peuvent favoriser la réduction des inégalités entre les filles et les garçons (Gutierrez \& GarciaLopez, 2012). Le développement d'une identité de classe et le passage d'un collectif d'élèves solitaires à un collectif d'élèves solidaires peuvent ainsi représenter une piste particulièrement intéressante en EPS (Bréau, 2013 ; Crance, 2015 ; Saury et al, 2013).

$\mathrm{Au}$ sein de cette étude, des limites doivent être soulignées aussi bien au niveau de l'analyse des résultats, que vis à vis de l'utilisation des focus groups comme unique outil d'investigation. Si les résultats présentés s'attachent à décrire le point de vue des adolescents à partir de différentes variables (niveau de scolarité, niveau de réussite en EPS), il aurait également pu être intéressant de poser la question de l'origine sociale des élèves (e.g., Lentillon, 2009b). Ce type de données reste néanmoins difficiles à obtenir dans le cadre d'études en milieu scolaire. Au regard de notre étude et du nombre relativement limité d'élèves interrogés qui considèrent avoir un niveau faible en EPS, il semble aussi important de poursuivre un travail autour des expériences vécues par des adolescents moins en réussite et de leur rapport vis à vis de la mixité et de la non mixité 


\section{eJRIEPS 40 janvier 2017}

en EPS. A ce titre, la mise en place dans cette étude d'une mesure de compétence autorapportée (et les possibles biais de sous ou sur-évaluation) peut être vue comme une limite. A propos des focus groups comme outil de recueil de données, bien que favorisant l'émergence de discussions riches, des entretiens individuels permettraient de préciser les expériences évoquées par les élèves, et notamment celles vécues dans d'autres activités que les seuls sports d'opposition, souvent évoqués par les adolescents dans cette étude. Afin d'enrichir ces données qualitatives, de futures recherches centrées sur l'analyse de l'activité des élèves en contextes mixte et non mixte semble être une piste non négligeable visant une connaissance plus fine et plus précise. A l'instar de travaux déjà réalisés qui privilégient une approche empirique (e.g., Amade-Escot, Elandoulsi \& Verscheure, 2015 ; Verscheure, Deforêt \& Poggi, 2014 ; Vinson \& Amade-Escot, 2014), la mise en place d'un dispositif d'observation in situ visant à repérer comment les élèves agissent et interagissent dans ces deux dispositifs permettrait ainsi de mieux comprendre la dynamique du genre, sa construction ou sa déconstruction au sein d'une leçon, d'un cycle ou d'une année scolaire. En EPS, des études ethnographiques (e.g., Berg \& Lahelma, 2010 ; Bréau, Hauw \& Lentillon-Kaestner, 2016a, 2016b ; Hill, 2015) ont notamment envisagé la construction du genre autour de la théorie du « doing gender » (West \& Zimmerman, 1987, 2009). Considérant le genre comme un « faire situé » ou une propriété émergente en cours de réalisation, la théorie du « doing gender » souligne la richesse et la pertinence des dispositifs d'observation. Plus proche de la réalité des pratiques, cette perspective pourrait également rendre compte de l'action et de l'intervention des enseignants, peu évoquées dans les focus groups.

\section{Conclusion}

A l'heure où la question de la non mixité à l'école continue d'occuper une place importante au sein des débats sur l'éducation, cette étude met en exergue les préférences d'une majorité de garçons, notamment scolarisés en secondaire 1 et affichant un niveau de réussite en EPS plutôt élevé, pour des cours séparés en classe d'EPS. En contexte non mixte, les garçons ont souligné la possibilité de s'engager pleinement dans une pratique compétitive, où le niveau d'apprentissage est meilleur qu'en contexte mixte. Si la non mixité est valorisée, des arguments avancés par des adolescents moins en réussite en EPS nous encouragent toutefois à poursuivre une réflexion à propos de la mise en place d'une pratique séparée en EPS. La non mixité interroge la prise en compte de la singularité de chaque élève, de chaque fille et de chaque garçon. Aujourd'hui, de fortes 


\section{eJRIEPS 40 janvier 2017}

critiques apparaissent contre la séparation des sexes à l'école, susceptible de négliger les différences et le genre de chaque adolescent (Jackson, 2010) et au sein de laquelle risque de se développer une «gender box» (Piatt, 2009) blessante pour celles et ceux qui peuvent présenter des caractéristiques généralement associées à l'autre sexe. A propos d'une EPS non mixte, les cours entre garçons doivent ainsi s'accompagner d'une réflexion autour du modèle de masculinité que cette discipline scolaire souhaite véhiculer. Un travail autour de la lutte contre les stéréotypes de genre semble également nécessaire afin de ne pas renforcer la division et l'opposition entre la masculinité et la féminité. Le développement d'une «compétence de genre » (Liebig, Rosenkranz-Fakkergger \& Meyerhofer, 2009) au sein de la formation initiale et continue des enseignants représente une piste d'action indispensable pour la construction de pratiques professionnelles favorisant la fluidité et la singularité des constructions identitaires des adolescents.

\section{Bibliographie}

Amade-Escot, C., Elandoulsi, S., \& Verscheure, I. (2015). Physical education in Tunisia : teachers' practical espistemology, students positionning and gender issues. Sport, education and society, 5, 656-675.

Auduc, J. L. (2009). Sauvons les garçons ! Paris, Editions Descartes.

Ayral, S., \& Raibaud, Y. (2014). Pour en finir avec la fabrique des garçons. Pessac: Ed. Maisons des sciences de l'homme d'Aquitaine.

Azzarito, L., Solmon, M. A., \& Harrison, J. (2006). «If I had a choice, I would... » A feminist poststructuralist perspective on girls in physical education. Research quarterly for exercise and sport, 77, 222-239.

Berg, P., \& Lahelma, E. (2010). Gendering processes in the field of physical education. Gender and educacion, 22, 31-46.

Best, S., Pearson, P. J., \& Webb, P. I. (2010). Teacher's perceptions of the effects of single sex and coeducacional classroom setting on the participation and performance of students in practical physical educacion. In A. Rendimimiento. (Ed.), Congreso de la asociacion internacional de escuelas superiores de educacion fisica (pp. 1016-1027). University of Wollongong.

Bigler, R. S., Hayes, A. R., \& Liben, L. S. (2014). Analysis and evaluation of the rationales for single-sex schooling. In L. S. Liben \& R. S. Bigler (Eds.), Advances in child development and behavior (pp. 225-260). Burlington : Academic Press. 


\section{eJRIEPS 40 janvier 2017}

Bohler, H. (2004). Sport education: examining an alternative Physical Educational instructional approach. Thesis in exercise and sports sciences. Texas Tech University, United States of America.

Bramham, P. (2003). Boys, masculinities and PE. Sport, education and society, 8, 57-71.

Bréau, A. (2013). Quand la classe devient une équipe ou quand «JE » est remplacé par « NOUS ». https://apprendreeneps.wordpress.com/2013/10/11/quand-la-classedevient-une-equipe-ou-quand-je-est-remplace-par-nous/

Bréau, A., Hauw, D., \& Lentillon-Kaestner, V. (2016a). Jeu «facile » vs « vrai » jeu en EPS. «Doing gender» et analyse de l'activité située de garçons au sein de classes mixtes et non mixtes. Poster présenté lors de la journée de la recherche Sciences Sociales et Politiques, 13 octobre 2016, Lausanne

Bréau, A., Hauw, D., \& Lentillon-Kaestner, V. (2016b). Analyse de l'activité des élèves et de la construction du genre au sein de classes non mixtes en éducation physique et sportive. Communication présentée lors du colloque international, «Penser les nouvelles problématiques éducatives dans une perspective internationale », 18 novembre 2016, Créteil.

Castillo-Andrès, O., Romera-Granados, S., Gonzalez-Ramirez, T. \& Campos-Mesa, M. (2012). Gender Equity in Physical Education : the use of information. Sex Roles, $67,108-121$.

Cazorla, G. (2011). Développement biologique et capacités physiologiques. In L. Lafont (Ed.), L'adolescence (pp. 49-68). Paris : Editions EPS.

Clément, X., \& Louveau, C. (2013). Socialisation sportive et formation des « Grands hommes ». Le cas du handball. Ethnologie française, 43, 723-733.

Cogérino, G. (2005). Filles et garçons en EPS. Paris : Editions Revue EPS.

Cogérino, G. (2007). Propos d'enseignants d'éducation physique face à la mixité. Revue Staps, 75, 25-42.

Combaz, G. (1991). La mixité en EPS : opinions et souhaits des élèves. Revue EPS, 231, 62-65.

Combaz, G., \& Hoibian, O. (2008). Le rôle de l'école dans la construction des inégalités de sexe. L'exemple de l'éducation physique et sportive. Travail, genre et sociétés, 20, 129-150.

Connell, R. W. (1999). Cool, guys, swots and wimps: the interplay of masculinity and education. Oxford review education, 15, 291-303.

Connell, R. W. (2002). Gender. Cambridge : Polity Press. 


\section{eJRIEPS 40 janvier 2017}

Connell, R. W., \& Messerschmidt, J. W. (2005). Hegemonic masculinity, rethinking the concept. Gender and society, 19, 829-859.

Couchot, Schiex, S., \& Trottin, B. (2005). Interactions enseignants/élèves : variations en fonction du sexe et du genre. In G. Cogérino (Ed.), Filles et garçons en EPS (pp. 163-182). Paris : Revue EPS.

Couchot-Schiex, S., \& Deriaz, B. (2013). Egalité en classes mixtes en éducation physique : quels arrangements pour les enseignant-e-s ? Revue des HEP et des institutions assimilées de Suisse romande et du Tessin, 16, 163-182.

Crance, M. C. (2015). Créer un spectacle de danse au collège : une aventure collective. In O. Vors (Ed.), L'activité collective (pp. 103-122). Paris : Revue EPS.

Crawford-Ferre, H. G., \& Wiest, L. R. (2013). Single sex educacion in public school settings. The educational forum, 77, 300-314.

Davisse, A. (1999). «Elles papotent, ils gigotent ». L'indésirable différence de sexes. Ville, école, intégration, 116, 185-198.

Dubet, F. (2010). L'école « embarrassée » par la mixité. Revue française de pédagogie, $171,77-86$.

Dubet, F., \& Martucelli, D. (1999). A l'école. Sociologie de l'expérience scolaire. Paris: Seuil.

Dumith, S. C., Gigante, D. P., Domingues, M. R., \& Kohl, H. W. (2011). Physical activity change during adolescence: a systematic review and a pooled analysis. International journal of epidemiology, 40, 685-698.

Duret, P. (1999). Les jeunes et l'identité masculine. Paris: Presses universitaires de France.

Fagrell, B., Larsson, H., \& Redelius, K. (2012). The game within the game : girl's underperforming position in Physical Education. Gender and education, 24, 101118.

Fassa, F. (2013). L'éducation à l'égalité entre les sexes dans l'école romande, une question vive ou inerte ? Revue des HEP et des institutions assimilées de Suisse Romande et du Tessin, 16, 13-29

Fergus, E., Noguera, P., \& Martin, M. (2014). Schooling for resilience : improving the life trajectory of Black and Latino boys. Cambridge : Harvard Education Press.

Fize, M. (2003). Les pièges de la mixité scolaire. Paris : Presses de la Renaissance.

Fraisse, G. (1996). La différence des sexes. Paris : PUF. 


\section{eJRIEPS 40 janvier 2017}

Gagné, G., Martel, D., \& Nadeau, L. (2006). Raisons évoquées par des élèves pour justifier leur préférence en matière de mixité ou de non mixité en éducation physique. In G. Carlier, D. Bouthier, \& G. Bui-Xuân. (Eds.), Intervenir en éducation physique et en sport : recherches actuelles (pp. 378-385). Belgique : PUL.

Garcia, C. (2000). Cet « autre » inaccessible. Corps et culture, 5, 1-13.

Godelier, M. (1996). La production des grands hommes. Paris : Fayard.

Grossenbacher, S. (2006). Vers l'égalité des sexes à l'école. Que font les cantons pour instaurer l'équité entre hommes et femmes dans le système éducatif ? Rapport de tendances. Aarau : Centre suisse de coordination pour la recherche en éducation.

Guérandel, C. (2013). L'apprentissage de la danse en collège ZEP à l'épreuve du genre. Revue Staps, 102, 31-46.

Guérandel, C., \& Beyria, F. (2010). La mixité dans les cours d'EPS d'un collège en ZEP : entre distance et rapprochement des sexes. Revue française de pédagogie, 170, 17-30.

Gurian, M., Stevens, K., \& Daniels, P. (2009). Successful single-sex classrooms : a practical guide to teaching boys and girls separately. San Francisco, CA : JosseyBass.

Gutierrez, D., \& García-López, L. M. (2012) Gender differences in game behaviour in invasion games. Physical Education and Sport Pedagogy, 17, 289-301.

Hannon, J., \& Williams, S. M. (2008). Should secondary physical education be coeducational or single-sex ? Journal of physical education, recreation \& dance, 79, 6-56.

Hardin, M., \& Greer, J. D. (2009). The influence of gender role socialization, media use, and sports participation on perceptions of gender appropriate sports. Journal of sport behavior, 32, 207-226.

Haywood, C., \& Mac an Ghaill, M. (2012). What's next for masculinity ? Reflexive directions for theory and research on masculinity and education. Gender and education, 24, 577-592.

Hickey, C. (2008). Physical education, sport and hyper-masculinity in schools. Sport, Education and Society, 13, 147-167.

Hill, J. (2015). Girl's active identities : navigating othering discourses of feminity, bodies and physical education. Gender and education, 27, 666-684. 


\section{eJRIEPS 40 janvier 2017}

Hills, L. A., \& Croston, A. (2012). It should be better all together : exploring strategies for « undoing » gender in coeducational physical education. Sport, education and society, 17, 591-605.

Jackson, J. (2010). «Dangerous presumptions» : how single-sex schooling reifies false notions of sex, gender and sexuality. Gender and education, 22, 227-238.

Kitzinger, J., Markova, I., \& Kalampalikis, N. (2004). Qu'est ce que les focus groups. Bulletin de psychologie, 47, 237-243.

Koca, C. (2009). Gender interactions in coed physical education : a study in Turkey. Adolescence, 44, 165-185.

Léchenet, A. (2014). Adapter l'école aux garçons ou lutter contre les stéréotypes. Travail, genre et sociétés, 31, 163-167.

Lentillon, V. (2009a). Les stéréotypes sexués relatifs à la pratique des activités physiques et sportives chez les adolescents français et leurs conséquences discriminatoires. Bulletin de psychologie, 62, $15-28$.

Lentillon, V. (2009b). La mixité en éducation physique et sportive : points de vue d'élèves du second degré. Revue eJRIEPS, 16, 38-54.

Lentillon, V., \& Cogérino, G. (2005). Les inégalités entre les sexes dans l'évaluation en EPS : sentiment d'injustice chez les collégiens. Revue STAPS, 68, 79-95.

Liebig, B., Rosenkranz-Fallegger, E., Meyerhofer, U. (2009). Manuel de compétences dans le domaine du genre. Guide pratique pour les universités et les hautes écoles spécialisées. Zurich : VDF.

Lyu, M., \& Gill, D. L. (2011). Perceived physical competence, enjoyment and effort in same sex and coeducational physical education classes. Educational psychology: an international journal of experimental education psychology, 31, 247-260.

Martino, W., \& Berrill, D. (2003). Boys, schooling and masculinities : interrogating the right way to educate boys. Educational review, 55, 99-117

Morgan, M., Gibbs, S., Maxwell, K. \& Britten, N. (2002). Hearing children's voices: methodological issues in conducting focus groups with children aged 7-11 years. Qualitatives research, 25, 5-12.

Mucchielli, R. (1998). L'analyse de contenu: des documents et des communications. Paris : ESF.

Ollis, D., \& Meldrum, K. (2009). Girls talking about girls' issues : the importance of girls only health and physical education in promoting well-being. Victoria: Deakin University. 


\section{eJRIEPS 40 janvier 2017}

Patinet-Bienaimé, C., \& Cogérino, G. (2011). La vigilance des enseignant-e-s d'éducation physique et sportive relative à l'égalité des filles et des garçons. Questions vives, 8, 1-13.

Piatt, B. (2009). Gender segregation in the public schools: opportunity, inequality or both ? Scholar, 11, 561-575.

Rapport Eurydice. (2010). Gender differences in educacional outcomes: study on the measures taken and the current situation in Europe. European Commission, Educacion, Audiovisual and Culture Executive Agency.

Rǿnholt, H. (2002). « It's only the sissies... » analysis of teaching and learning process in physical educacion : a contribution to the hidden curriculum. Sport, educacion and society, 7, 25-36.

Rouyer, V. (2007). La construction de l'identité sexuée. Paris : Armand Colin.

Saury, J., Adé, D., Gal Petifaux, N., Huet, B., Sève, C., \& Trohel, J. (2013). Actions, significations et apprentissages en EPS. Paris : Editions EPS.

Smyth, E. (2010). Single-sex education: what does research tell us ? Revue française de pédagogie, 171, 47-55.

Swain, J. (2006). Reflections on patterns of masculinity in school settings. Men and masculinities, 8, 331-349.

Thorne, B. (1993). Gender play. Girls and boys in school. Buckingham : Open University Press.

Tischler, A., \& McCaughtry, N. (2011). PE is not for me. Research quarterly for exercise and sport, 82, 37-48.

Treanor, L., Graber, K., Housner, L., \& Wiegand, R. (1998). Middle schools students' perceptions of coeducational and same-sex physical education classes. Journal of teaching in physical education, 18, 43-56.

Verscheure, I., Deforêt, L., \& Poggi, M. P. (2014). Lorsque les inégalités de genre se construisent en classe: analyse d'une situation d'apprentissage visant l'équité pour accompagner le changement des pratiques. Communication présentée lors du congrès ARIS, Temps, temporalités et intervention en EPS et en sport, Genève.

Vinson, M., \& Amade-Escot, C. (2014). Sous la dynamique non verbales des interactions didactiques, «l'impensable du genre ». In I. Collet., \& C. Dayer (Eds.), Former envers et contre le genre, (pp. 219-246). Louvain-la-Neuve : De Boeck 


\section{eJRIEPS 40 janvier 2017}

Vouillot, F. (2010). La mixité, une évidence trompeuse ? Entretien avec Martine Chaponnière, Université de Genève. Revue française de pédagogie, 171, 69-75.

Welzer-Lang, D. (2000). Pour une approche pro-féministe non homophobe des hommes et du masculin. In D. Welzer-Lang (Ed.), Nouvelles approches des hommes et du masculin, (pp. 109-138). Toulouse : Presses universitaires du Mirail.

Welzer-Lang, D. (2010). La mixité non ségrégative confrontée aux constructions sociales du masculin. Revue française de pédagogie, 171, 15-29.

Welzer-Lang, D., \& Zaouche-Gaudron, C. (2011). Masculinités : état des lieux. Toulouse : Erès.

West, C., \& Zimmerman, D. H. (1987). Doing gender. Gender and society, 1, 125-151.

West, C., \& Zimmerman, D. H. (2009). Accouting for doing gender. Gender and society, 23, 112-122.

With-Nielsen, N., \& Pfister, G. (2011). Gender constructions and negotiations in physical education : case studies. Sport, educacion and society, 16, 645-664. 